\title{
Cystic medial degeneration of the aorta is associated with p53 accumulation, Bax upregulation, apoptotic cell death, and cell proliferation
}

C Ihling, T Szombathy, K Nampoothiri, J Haendeler, F Beyersdorf, M Uhl, A M Zeiher, H E Schaefer

Abstract
Objective-To address a potential role for p53, Bc12 associated protein $X$ (Bax), and apoptosis in the processes associated with cell turnover during cystic medial degeneration (CMD) of the aorta.

Methods-Histochemical, immunohistochemical, biochemical, and morphometric methods were used to assess the presence and distribution of p53 immunoreactivity (p53-IR) and Bax immunoreactivity (Bax-IR), as well as the presence of apoptosis and tissue repair processes.

Results-Immunohistochemical staining disclosed evidence for p53-IR in all specimens in 26.1 (11.5)\% of vascular smooth muscle cells (VSMCs) (controls 0.8 (1.3)\%; p < 0.001). Bax-IR was present in all specimens in $10(5.4) \%$ of medial cells (controls $0.3(0.5) \% ; p<0.001)$. Medial VSMCs ( $\alpha$-actin positive) with cytoplasmic staining for an apoptosis specific protein (c-jun/ASP) were present in 20/20 specimens $(0.7(0.6) \%$ of VSMCs, controls $0 \%, p<0.001$, whereas terminal deoxynucleotidyl transferase mediated dUTPbiotin nick end labeling (TUNEL) positive VSMCs were present in $17 / 20$ specimens (1 (1.5)\% of VSMCs, controls $0 \%, p<0.001)$. The presence of apoptosis was confirmed by electron microscopy and the demonstration of oligonucleosomal DNA fragments after agarose gel electrophoresis. As shown by double labeling and investigation of serial sections, p53-IR, Bax-IR, c-jun/ASP-IR, and positive TUNEL labeling localised to the same compartments of the aortic media, raising a possible role for p53 and Bax in the triggering of apoptosis of VSMC during CMD. MIB1/Ki-67 positive medial VSMCs ( $\alpha$-actin positive) and mesenchymal cells (vimentin positive) were present in all specimens $(2.5$ (2.8)\% of medial cells; controls $0.3(0.9) \%$, $\mathrm{p}<0.001)$ mainly in the region around the vasa vasorum, indicating that cell regeneration during CMD may originate mainly from the mesenchyme surrounding the vasa vasorum.

Conclusion-This study shows that the formal pathogenesis of CMD is characterised by p53 accumulation, Bax upregulation, cell death by apoptosis, and cell regeneration. Nevertheless, the precise stimuli of p53 activation and Bax upregu- lation as well as the role of p53 and apoptosis in the dissection process itself remain elusive.

(Heart 1999;82:286-293)

Keywords: mucoid cystic medial degeneration of the aorta; 553 accumulation; apoptosis; cell proliferation

Thoracic aortic aneurysms and aortic dissection have been reported in association with a variety of predisposing factors, including genetic disorders and hypertension. ${ }^{12}$ In many cases, the media shows characteristic degenerative changes of vascular smooth muscle cells (VSMCs), the extracellular matrix, and of the elastic tissue called cystic medial degeneration (CMD). CMD is a non-inflammatory, age related, ${ }^{34}$ non-specific intrinsic reaction pattern of the aortic media, which is typically accompanied by elastin fragmentation, focal fibrosis, accumulation of acid mucopolysaccharides to a variable extent, haphazardly distributed areas with loss of VSMCs, and accumulation of collagen. The focal absence of medial VSMCs without any concomitant inflammation is highly suggestive of cell loss mediated by apoptotic processes.

In contrast to "oncosis" or "accidental cell death", "apoptosis" or "programmed cell death" is an active form of cell death, which is triggered by a large amount of distinct death stimuli, transduced through numerous signalling mechanisms finally leading to the activation of the caspase cascade, which results in a systematic degradation of cellular proteins and DNA. The morphological substrates of this breakdown are chromatin condensation and margination, nuclear fragmentation, and membrane budding. ${ }^{56}$ Programmed cell death is regulated through different mechanisms including the expression of the members of the Bcl2 protein family consisting of pro- and antiapoptotic peptides interacting with each other by forming homo- and heterodimers. The $21 \mathrm{kD}$ protein of the $\mathrm{Bcl} 2$ protein family "Bcl2 associated protein X" (Bax) counteracts the antiapoptotic effects of several members of this protein family and promotes apoptosis when overexpressed. ${ }^{7}$ It has been shown that the protein product of the wild-type tumour suppressor gene p53 (wt p53) may upregulate the transcription of the Bax gene $\mathrm{e}^{8}$ and repress the transcription of the antiapoptotic $\mathrm{Bcl} 2$ gene. ${ }^{9}$ Moreover, p53 is involved in the 
regulation of cell cycle checkpoints, ${ }^{10}$ cell differentiation, ${ }^{11}$ in the response to certain cellular stress signals, ${ }^{12}$ as well as in the triggering of apoptosis following DNA damage. ${ }^{13}$ Also, p53 has been implicated in VSMC apoptosis in vitro $^{14}$ and in vivo, ${ }^{15}$ and in the control of the proliferation of human VSMCs. ${ }^{16}{ }^{17}$ Furthermore, a role for p53 has also been demonstrated in association with wound healing, indicating a significant role for $\mathrm{p} 53$ in the processes associated with tissue injury and repair. ${ }^{18-20}$

CMD has been considered as a process of tissue injury and repair following mechanical traumas caused by haemodynamic forces. ${ }^{34}$ It typically leads to tissue inhomogeneity and thus potentially causes abnormal levels of shear stress acting on the myocytes embedded in the extracellular matrix. ${ }^{21}$ Importantly, recent observations indicate that following mechanical stretching of cardiac cells p53 is activated, Bax is upregulated in a p53 dependent fashion, and apoptosis increases. ${ }^{22}$ Thus, in order to address a potential role for Bax, p53, and programmed cell death in the processes associated with cell turnover during CMD of the aorta, we assessed the presence and distribution of Bax immunoreactivity (Bax-IR) and p53 immunoreactivity (p53-IR), as well as the presence of apoptosis and tissue regeneration, using histochemical, immunohistochemical, biochemical, and morphometric methods.

\section{Methods}

TISSUE SPECIMENS

Full thickness aortic wall specimens from 20 patients (three female, 17 male; mean (SD) age 49.37 (18.03) years) with spontaneous aortic rupture and without phenotypic characteristics of any of the known genetic disorders, such as Marfan's disease, were obtained after surgery. The plane of dissection was invariably located in the outer third of the media. Control experiments were performed by staining punch biopsies of the ascending aorta from 10 patients (three female, seven male; mean age 58.7 (5.39) years) undergoing aortocoronary bypass surgery. The specimens were fixed in $4 \%$ unbuffered formalin, embedded in paraffin, and then prepared according to standard methods. Serial sections were stained with haematoxylin and eosin (H\&E), elastica van Gieson, and alcian blue for the detection of acid mucopolysaccharides, and used for histochemistry and immunohistochemistry. For biochemical DNA fragmentation analysis, aortic tissue from a patient undergoing resection of an aneurysm of the ascending aorta without dissection as well as without any visible atherosclerotic lesions, and from a control patient without aortic disease, were snap frozen in liquid nitrogen.

TUNEL

Terminal deoxynucleotidyl transferase (TdT) mediated dUTP-biotin nick end labeling (TUNEL) for detecting DNA breaks in situ was performed essentially as described by Kockx. ${ }^{23}$ In brief, after the quenching of endogenous peroxidase, sections were rinsed in
TdT buffer ( $30 \mathrm{mM}$ TRIS, $140 \mathrm{mM}$ sodium cacodylate, $1 \mathrm{mM}$ cobalt chloride) at $\mathrm{pH} 7.2$ and incubated with $0.3 \mathrm{eu} / \mathrm{ml}$ TdT (Sigma, München, Germany) and biotinylated-dUTP (1:200, Boehringer, Mannheim, Germany) in TdT buffer for 60 minutes at $37^{\circ} \mathrm{C}$. Labelled nuclei were detected with Vectastain ABC (Vector Labs, Burlingame, California, USA) and peroxidase activity was visualised by 3-amino-9-ethylcarbazole (AEC) to yield a reddish brown reaction product. The sections were lightly counterstained with haematoxylin. As a positive control, tissue sections of follicular hyperplasia of the appendix were used and gave the expected positive staining by TUNEL of tingible bodies in the germinal centres. To identify VSMCs and cells expressing ASP/cjun showing a positive TUNEL stain, double labeling was performed with an anti- $\alpha$-actin antibody (HHF 35, DAKO, Hamburg, Germany) and the anti-ASP/c-jun antibody (see below). In brief, TUNEL staining was performed using the above described method visualising peroxidase activity with AEC which resulted in a reddish brown staining. In a second staining procedure, immunohistochemistry was performed using the three step APAAP method. Activity of alkaline phosphatase was visualised with naphthol AS-MX phosphate (Sigma) and fast blue BB salt (Sigma) substrate solution which resulted in a blue staining. Endogenous alkaline phosphatase was blocked by adding $0.01 \mathrm{ml}$ of $1 \mathrm{M}$ levamisole to the substrate solution. The presence of both reaction products was identified by a distinctly separate brown staining of the nucleus and a dark blue staining of the cytoplasm.

\section{IMMUNOHISTOCHEMISTRY}

Immunohistochemistry was performed using either a modified three step avidin-biotin complex method as previously described ${ }^{16}{ }^{17}$ or the indirect tyramide signal amplification (DuPont, Boston, Massachusetts, USA p53) according to the manufacturer's instructions. The slides were incubated with primary antibodies directed against smooth muscle $\alpha$-actin (dilution 1:600; HHF 35, DAKO), p53 (clone DO 1, dilution 1:250, Oncogene Science Cambridge, USA) recognising both wild type as well as the mutant p53 protein, MIB1/Ki-67 (monoclonal, 1:50, Dianova Hamburg, Germany), Bax (polyclonal,1:100, $\mathrm{DAKO}$ ), and $\mathrm{ASP} / \mathrm{c}-\mathrm{jun}(\mathrm{Ab} 2$, dilution 1:10, Oncogene Science) against a synthetic peptide corresponding to residues 73-87 of v-jun for visualisation of apoptotic cells by detecting a cytoplasmic apoptosis specific protein (ASP), ${ }^{24}$ respectively. For immunostaining antigens were either unmasked by pressure cooking in $10 \mathrm{mmol}$ citric acid, $\mathrm{pH} 6$ for five minutes (p53, MIB1/Ki-67, Bax) or by partial digestion with proteinase $\mathrm{K}$ for five minutes (ASP/c-jun). As a positive control for p53 and smooth muscle $\alpha$-actin staining we used sections from a follicular hyperplasia of a tonsil with a well known immunoreactivity for the antigens, and as a positive control for Bax and ASP/c-jun we stained human atherosclerotic carotid plaques 

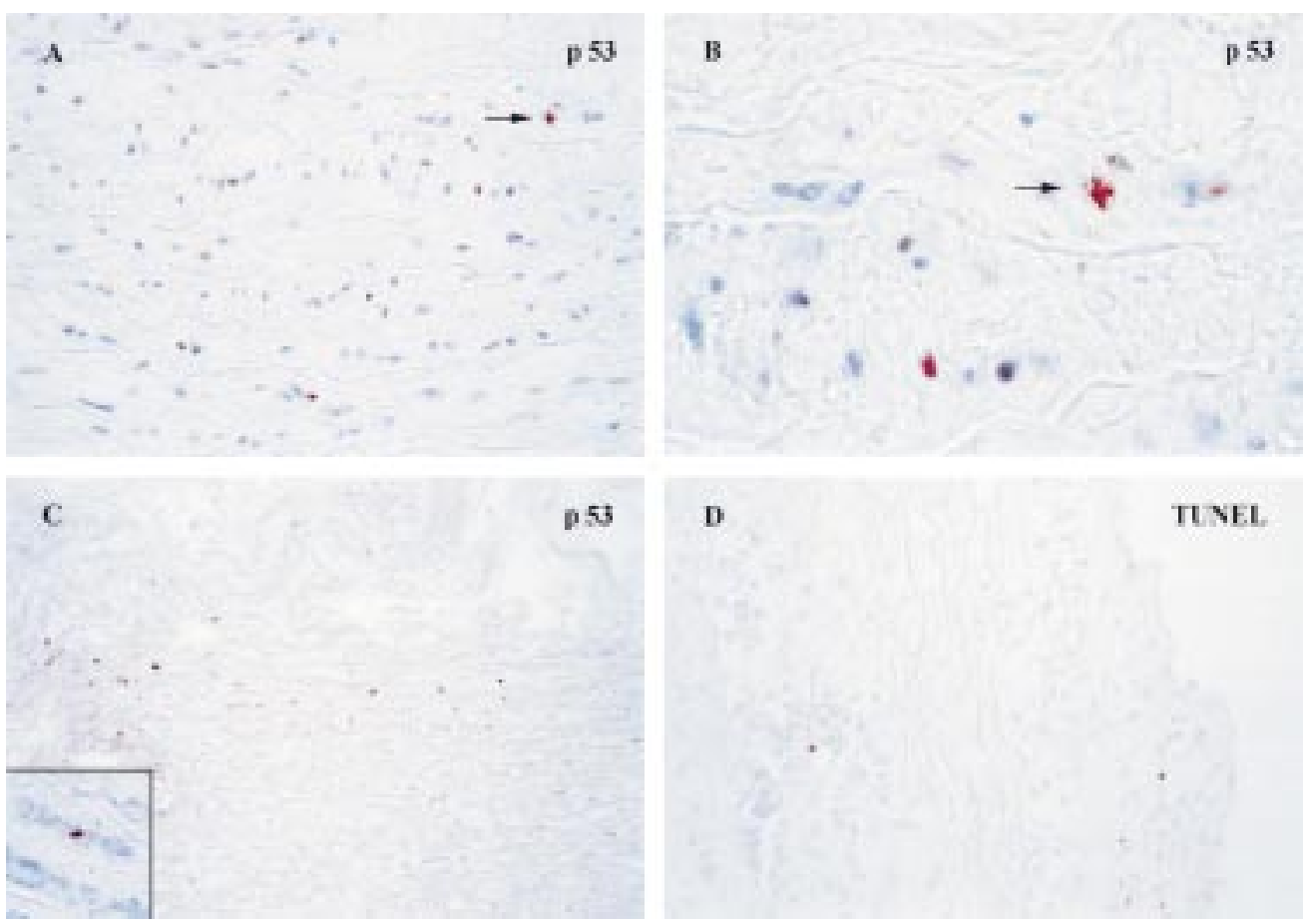

D

TINEL.

Figure 1 (A) Low power magnification from a p53 stained section of a segment of a human aorta with CMD. Shown is an area at some distance from the dissection plane with only minor degrees of structural changes. There are numerous nucle in a haphazard distribution displaying a positive staining for $p 53$ (brown reaction product in the nuclei). (B) Higher power magnification of the region which is marked by an arrow on A. Note the $p 53$ positive nucleus indicated by the arrow displays the typical nuclear morphology of apoptosis-for example, chromatin condensation and fragmentation. (C) Low power magnification of a p53 stained section of a segment of a human aorta with CMD showing the plane of dissection (upper half) and its immediate vicinity (lower half). There are numerous $p 53$ positive nuclei in a homogenous distribution. Inset: double immunostaining for $p 53$ (brown reaction product, $A B C$ method) and a-actin (blue reaction product, APAAP method) showing a medial VSMC with nuclear p53 accumulation. (D) Low power magnification of a TUNEL stained section of a segment of a human aorta with CMD showing the plane of dissection (right side). There are numerous nuclear fragments exhibiting a positive TUNEL stain located in the immediate vicinity of the dissection plane.

where c-jun staining and Bax staining is abundant. ${ }^{25} 26$ Negative control experiments were performed by replacing the primary antibodies with preimmune serum of the corresponding species (mouse, rabbit). Peroxidase activity was visualised by 3-amino-9ethylcarbazole (AEC, Sigma) to yield a reddish brown reaction product. The nuclei were lightly counterstained with haematoxylin. Double labeling to identify smooth muscle cells with p53 accumulation and MIB1/Ki-67 expression as well as medial cells with coexpression of p53 and Bax was performed combining the ABC method and the APAAP method as previously described. ${ }^{16}$ Briefly, the labeling procedure was as follows. First, p53 or MIB1/Ki-67 staining was performed using one of the above described ABC methods visualising peroxidase activity with AEC or 3,3'diaminobenzidine which resulted in a reddish brown or dark brown staining. After thorough washing, in a second staining procedure, $\alpha$-actin staining or Bax staining was performed using the three step APAAP method. Activity of alkaline phosphatase was visualised with naphthol AS-MX phosphate and fast blue BB salt or fast red TR salt (Sigma). The presence of both antigens was identified by a distinctly separate reddish brown (MIB1/Ki-67) or dark brown (p53) staining of the nuclei and blue $(\alpha$-actin) or red (Bax) staining of the cytoplasm.
HISTOLOGICAL ANALYSIS

The specimens were analysed by light microscopy for the presence and localisation of antibody mediated and terminal transferase mediated staining. Comparative examination of serial sections and double labeling permitted the assessment of colocalisation of $\mathrm{p} 53$ positive cells with cytoplasmic staining for $\alpha$-actin as well as for MIB1 positive cells with cytoplasmic staining for $\alpha$-actin.

MORPHOMETRIC ANALYSIS AND STATISTICAL METHODS

To study the total cell numbers per high power field, and to study quantitatively the distribution of cells with p53 accumulation, $\mathrm{MIB} 1 / \mathrm{Ki}-$ 67, Bax and c-jun/ASP expression and TUNEL positive nuclei adjacent sections from all 20 cases were evaluated. Using a morphometric software (analySIS, Softimaging Software), five random microscopic high power fields of the media at a distance from the dissection plane (at least five high power fields $=12.5 \mathrm{~mm}$ ) at $\times 400$ were analysed, scoring at least 500 cells as positive or negative for antibody mediated or terminal transferase mediated staining. All data are reported as mean (SD). The Mann-Whitney U test was employed for comparison of the aortic tissue showing mucoid degeneration with the controls. 

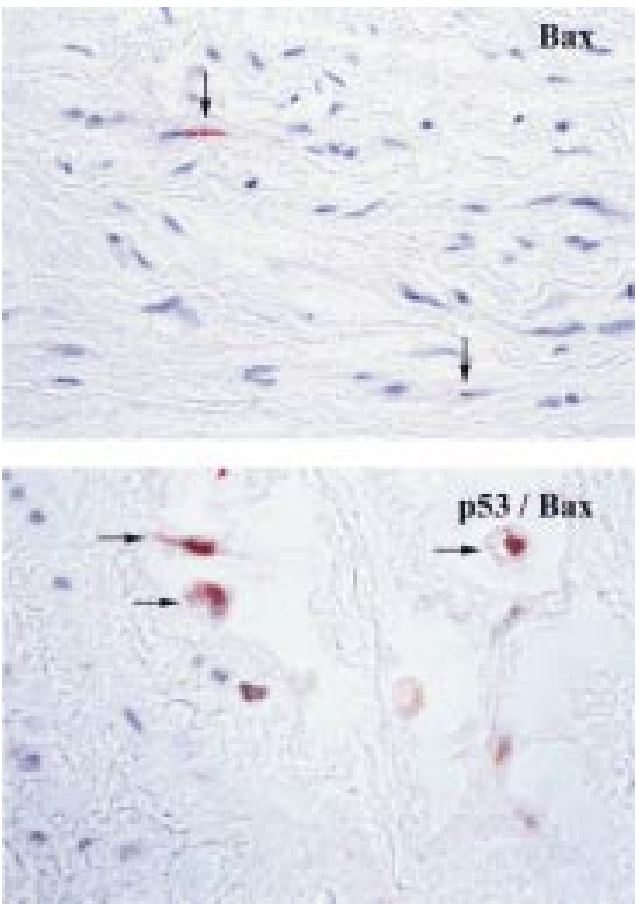

Figure 2 (Top) Low power magnification from a Bax stained section of human aorta with only minor degrees of CMD showing two medial cells with cytoplasmic Bax positivity (arrows, reddish brown reaction product). (B)

High power magnification from a Bax/p53 immuno double labeling showing an area with advanced CMD. In a region with pseudocystic degeneration there are three cells showing cytoplasmic Bax positivity (red reaction product) and simultaneous nuclear p53 staining (dark brown reaction product).

DNA ISOLATION AND DNA FRAGMENTATION ANALYSIS

The frozen tissue was immersed in a mortar with liquid nitrogen and ground with a pestle. Then, the frozen tissue powder was dissolved in $1 \mathrm{ml}$ of lysis buffer ( $5 \mathrm{mM}$ TRIS-HCl, pH 8.0; $20 \mathrm{mM}$ EDTA; 0.5\% Triton-X 100), lysed on ice for 30 minutes and subsequently incubated with $5 \mathrm{mg} / \mathrm{ml} \mathrm{RNase} \mathrm{A} \mathrm{at} 37^{\circ} \mathrm{C}$ for 30 minutes followed by a further incubation with $0.5 \mathrm{mg} /$ $\mathrm{ml}$ proteinase $\mathrm{K}$ and $0.1 \%$ sodium dodecylsulfate at $65^{\circ} \mathrm{C}$ for two hours. After phenol/ chloroform extraction, DNA was precipitated with $70 \%$ isopropanol and $0.1 \mathrm{M}$ sodium chloride. The resulting pellet was resolved in $\mathrm{TE}$ buffer (10 mM Tris-HCl, pH 8.0; $1 \mathrm{mM}$ EDTA). Then, $5 \mathrm{mg}$ DNA was treated with $5 \mathrm{U}$ of Klenow polymerase using $0.5 \mathrm{mCi}$ of $\left[{ }^{32} \mathrm{P}\right]$ labelled dCTP in the presence of $10 \mathrm{mM}$ Tris-HCl, pH 7.5 and $5 \mathrm{mM}$ magnesium chloride for 10 minutes. The reaction was stopped by adding $10 \mathrm{mM}$ EDTA, and the unincorporated nucleotides were removed by Sephadex G-50 columns (Pharmacia, Freiburg, Germany). DNA electrophoresis was carried out in a $1.8 \%$ agarose gel (three hours at $90 \mathrm{~V}$ ) containing $1 \mathrm{mg} / \mathrm{mL}$ ethidium bromide. Then DNA was transferred to nitrocellulose Hybond $\mathrm{N}+$ (Amersham, Braunschweig, Germany) and DNA fragments were visualised by exposing the membrane to radiographic film.

ELECTRON MICROSCOPY

Tissue samples were taken from an aortic specimen derived from a patient with an aneu-

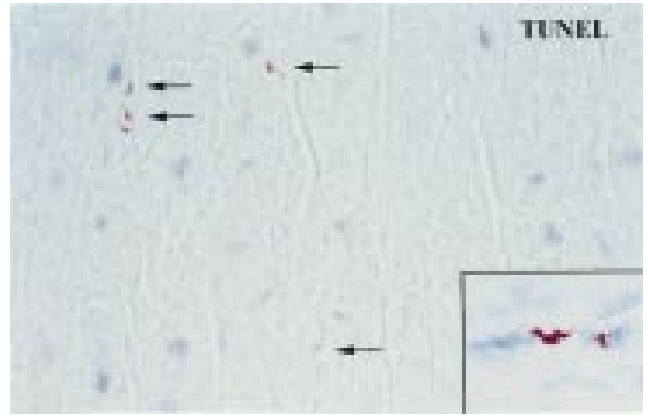

Figure 3 High power magnification from a TUNEL stained section of a segment of a human aorta with CMD at some distance from the dissection plane. There are several small TUNEL positive particles (arrows) probably corresponding to the apoptotic bodies shown in fig 6C. Inset: double labeling showing medial VSMCs with a positive TUNEL stain in the nucleus (brown reaction product) and cytoplasmic staining for a-actin (blue reaction product, APAAP method). One TUNEL positive nucleus shows the typical fragmentation which is a morphological feature of apoptosis.

rysm of the ascending aorta without dissection. The presence of CMD and absence of atherosclerotic changes was confirmed by macroscopic examination and light microscopy. Then, $1 \mathrm{~mm}$ cubes were fixed at room temperature in $3.5 \%$ glutaraldehyde in $0.1 \mathrm{~mol} / \mathrm{L}$ cacodylate buffer, washed in cacodylate buffer, dehydrated through graded ethanols and propyleneoxide, postfixed in 1\% osmium tetroxide, and embedded in epoxy resin. Thin sections were cut on a diamond knife and double stained with uranyl acetate and lead citrate before examination in a Philips 400 electron microscope.

\section{Results}

HISTOLOGY

All 20 tissue specimens showed the typical histopathological features of CMD in varying proportions and irregular patchy distribution. Lesion severity was classified according to the scheme of Schlattmann and Becker. ${ }^{3}$ The changes encompassed small cleft like or cystic spaces filled with alcian blue positive amorphous, acid mucopolysaccharides of grades I-III in $20 / 20$ cases, being most prominent around the vasa vasorum, elastin fragmentation of grades I-III in 20/20 cases, focal loss of nuclei of grade I in $20 / 20$ cases, and a mild increase in collagen content of grade I in 13/20 cases. Signs of inflammation in the media were absent in all cases. A fresh plane of dissection covered with thrombotic material was present in $15 / 20$ cases. In three cases, we found evidence of subacute dissection because the plane of dissection was covered with granulation tissue as a sign of wound healing and organising haematoma, and in two cases the plane of dissection was absent. Age related intimal fibrosis was present in $7 / 20$ cases, and intimal atherosclerotic lesions (Stary II-III) ${ }^{27}$ with only a minor degree of inflammatory activity, and without prominent atherosclerosis related alterations of the media beneath, were present in 13/20 cases. In the control tissue, we found mucoid CMD of grade $I$ in $7 / 10$ specimens, focal medial fibrosis with loss of nuclei in $1 / 10$ specimens, atherosclerotic 


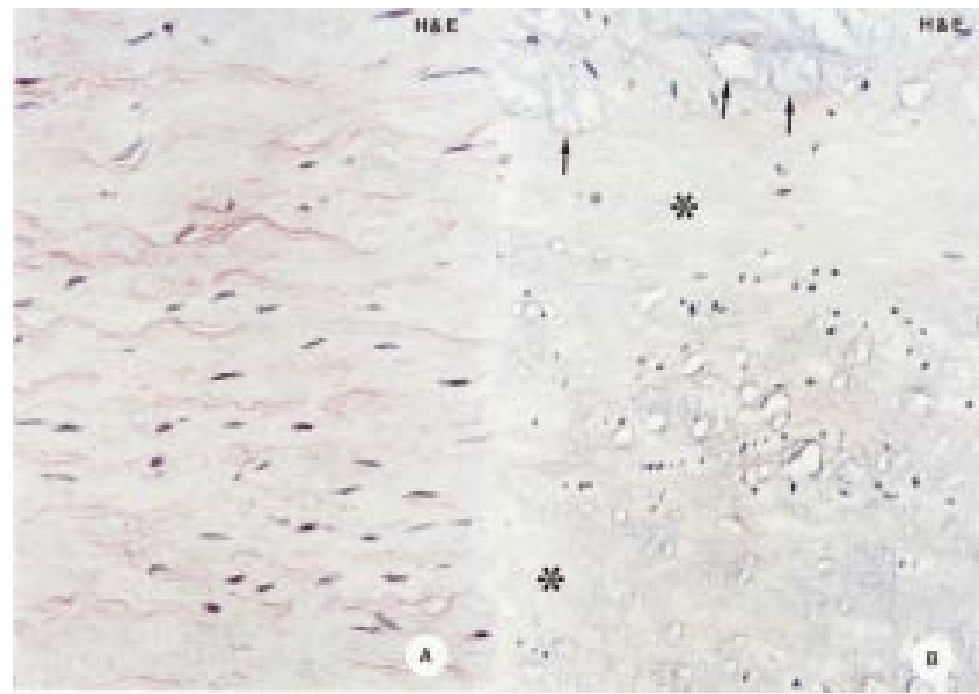

Figure 4 (A) HEE staining of a representative part of aortic control tissue used for DNA fragmentation analysis. There are no structural alterations in the media. The nuclei are oriented in a parallel fashion to the elastic fibres. (B) $H \mathcal{E} E$ staining of a representative part of the aortic tissue from the patient with an aneurysm of the ascending aorta used for $D N A$ fragmentation analysis showing the typical alterations of CMD-for example, small pseudocysts filled with basophilic amorphous material (arrows) and focal loss of nuclei (asterisks). Signs of inflammation in the media were absent. In addition, despite the focal absence of cell nuclei (asterisks), the total cell count in B is higher than in A.

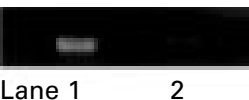

Lane 1 2

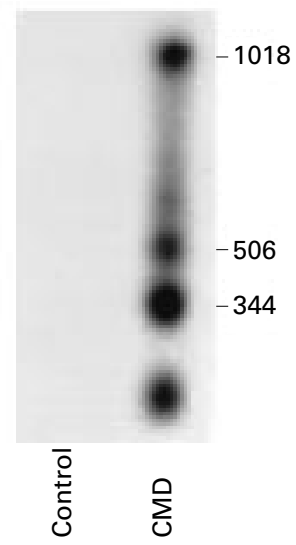

Figure 5 Agarose gel electrophoresis of DNA isolated from a control patient and a patient with CMD. Lane 1, control patient; lane 2, patient with $C M D$. In lane 2 there are oligonucleosomal DNA fragments indicating cell death by apoptosis. In contrast, in the control tissue oligonucleosomal DNA fragments were absent. Top of lane 1 - total amount of DNA brought into the gel. lesions of the type Starry I-II in 5/10 cases, and intimal fibrosis in 2/10 specimens, whereas two specimens did not show any morphological alterations.

\section{p53 IMMUNOHISTOCHEMISTRY}

Immunohistochemical staining disclosed evidence for p53 staining in 20/20 specimens. In the regions of the media which were not affected by the dissection (fig 1A, B), VSMCs $(\alpha$-actin positive, fig $1 \mathrm{C}$, inset) with nuclear p53 accumulation were distributed in a haphazard fashion. Furthermore, p53 positive mesenchymal cells (vimentin positive) were present in the regions surrounding the vasa vasorum. In addition, we found $\mathrm{p} 53$ positive medial VSMCs with the typical nuclear morphology of apoptosis - for example, chromatin condensation and fragmentation (fig 1A, $\mathrm{B}$, arrows). In contrast, in the immediate vicinity of the dissection plane p53 positive cells occurred in a homogenous distribution (fig 1C). The intensity of p53 staining varied considerably. However, most of the nuclei displayed a distinct and finely granular brown staining, which is a sign of p53 accumulation. Quantitative analysis revealed that 26.1 (11.5)\% of the medial cells exhibited nuclear accumulation of $\mathrm{p} 53$, whereas in the control group p53 staining was restricted to $0.8(1.3) \%$ of the medial cells $(p<0.001)$. In addition, few intimal cells showed p53 accumulation in $17 / 20$ cases.

Bax IMMUNOHISTOCHEMISTRY AND RELATION TO p53 ACCUMULATION

Immunohistochemistry revealed randomly distributed Bax positive medial cells (fig 2A) with VSMC like morphology in $20 / 20$ specimens with CMD and in $7 / 10$ control specimens (CMD 10 (5.4)\% of the medial cells; controls $0.3(0.5) \%, p<0.001)$, mainly in the regions where $\mathrm{CMD}$ was particularly pronounced as indicated by the presence of large pseudocystic spaces or disappearance of nuclear staining as a sign for loss of VSMCs. In addition, cytoplasmic Bax positivity was present in luminal endothelial cells, the endothelial cells of the vasa vasora, and the mesenchymal cells next to the vasa vasora. Similar to p53 staining, in the immediate vicinity of the dissection plane Bax positive cells occurred in a more homogeneous distribution. Furthermore, whenever present, intimal foam cells showed a finely granular Bax immunoreactivity. Double immunolabeling for p53 and Bax revealed the presence of $\mathrm{p} 53^{+} /$ $\mathrm{Bax}^{+}, \mathrm{p} 53^{+} / \mathrm{Bax}^{-}$(fig $2 \mathrm{~B}$ ) and $\mathrm{p} 53^{-} / \mathrm{Bax}^{+}$medial cells with VSMC like morphology.

\section{DNA NICK END LABELING, DNA FRAGMENTATION} ANALYSIS, AND ELECTRON MICROSCOPY

Aortic tissue specimens of $17 / 20$ patients demonstrated DNA strand breaks in the media, as shown by TUNEL assay. Nuclear fragments exhibiting a positive TUNEL stain were invariably located in the immediate vicinity of the dissection plane (12/17 cases, fig 1D). Furthermore, as confirmed by double labeling, at some distance of the dissection plane small TUNEL positive ( $\alpha$-actin negative) particles (fig 2 ) and TUNEL positive VSMCs were detected ( $\alpha-$ actin positive, inset fig 3 ) in the vicinity of the characteristic small cystic spaces and the spaces around the vasa vasorum $(1$ (1.5)\% of the cells, controls $0 \%, \mathrm{p}<0.001)$. To obtain biochemical evidence for apoptosis of VSMCs, we isolated genomic DNA from an aortic specimen derived from a patient with an aneurysm of the ascending aorta with CMD (fig $4 \mathrm{~B})$, in the absence of atherosclerosis as confirmed by histology, and analysed the size of the DNA fragments by agarose gel electrophoresis. After electrophoresis, we observed oligonucleosomal DNA fragments at about $180 \mathrm{bp}$ or multiples indicating cell death by apoptosis (fig 5, lane 2). In contrast, the histopathological alterations of CMD or oligonucleosomal DNA fragments were not observed in the control tissue (fig 4A, fig 5, lane 1). Furthermore, we demonstrated the presence of VSMC meeting the ultrastructural criteria of apoptosis - that is, margination and condensation of chromatin, membrane blebbing, and a convoluted cell surface (fig 6B), as well as the presence of numerous extracellular membrane bound apoptotic bodies containing nuclear remnants (fig 6C).

ASP/c-jun IMMUNOHISTOCHEMISTRY AND RELATION TO p53 ACCUMULATION

$\mathrm{ASP} / \mathrm{c}$-jun is a $45 \mathrm{kD}$ cytoplasmic protein which is recognised by a polyclonal antibody raised against a synthetic peptide corresponding to part of the amino acid sequence of c-jun. It is clearly distinct from jun and forms part of a modified cytoskeleton unique to preapoptotic cells and cells undergoing apoptosis. ASP/c-jun is present in cells which have activated their apoptotic programme, but not all of them have already reached an irreversible point. In contrast, ASP was not detected in necrotic cells. $^{24}$ ASP/c-jun-IR was present in 20/20 specimens (fig 7 ) in $0.7(0.6) \%$ of medial 


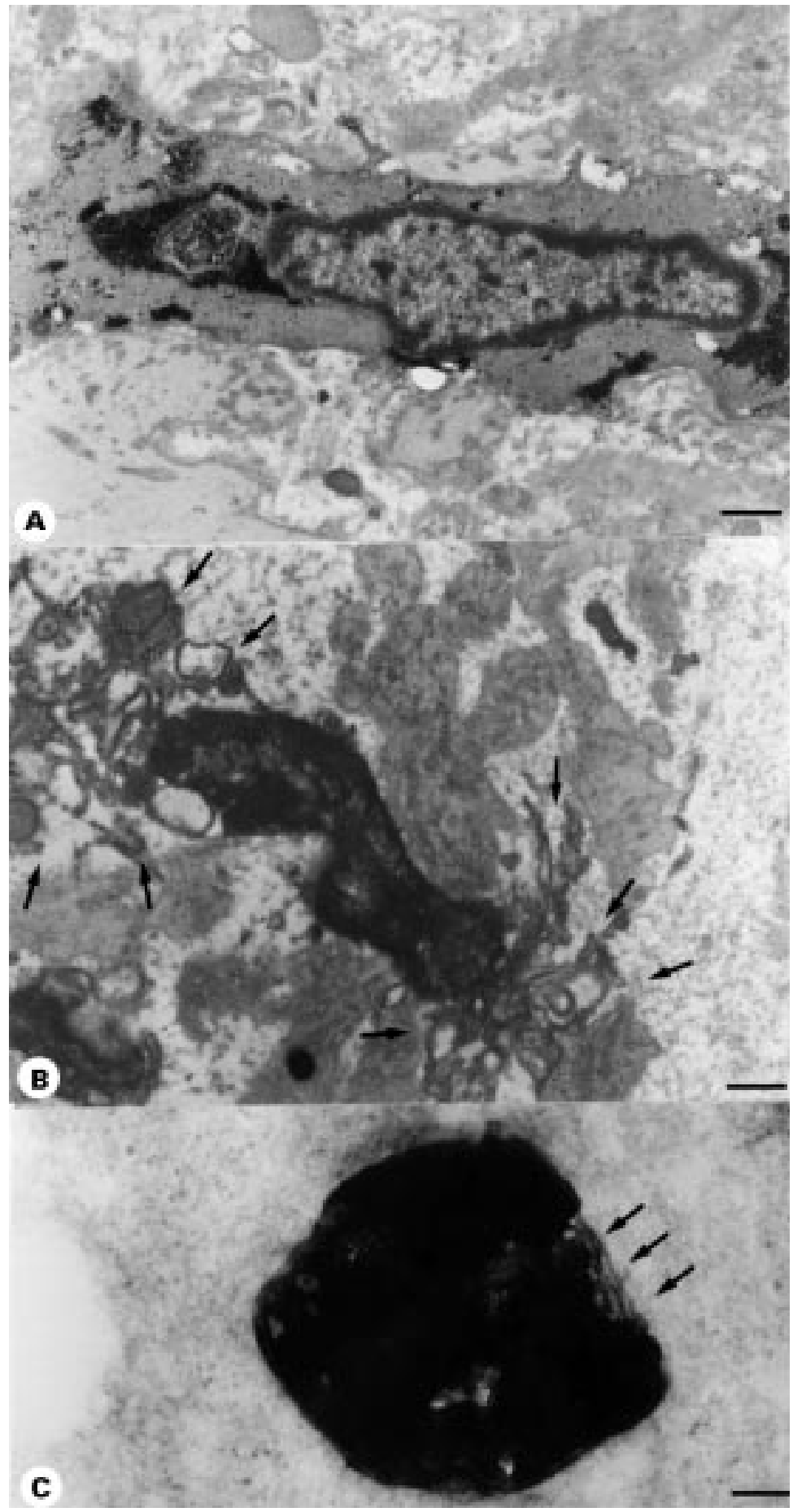

Figure 6 (A) Transmission electron micrograph of a normal VSCM from a patient with an aneurysm of the ascending thoracic aorta with CMD. Cellular organelles and the nucleus are intact and large amounts of glycogen are present. Scale bar $5 \mu \mathrm{m}$. (B)

Transmission electron micrograph of a VSMC from the same patient displaying the typical ultrastructural features of the early stages of apoptosis-for example, membrane blebbing (arrows), dissolution of the cytoplasm, as well as condensation and margination of the chromatin. For comparison no such changes are present in A. Scale bar $5 \mu \mathrm{m}$. (C) In the aortic media of the same patient numerous typical apoptotic bodies representing the late stage of programmed cell death were present. They contain an intensely osmophilic amorphous material probably representing a fragment of a condensed electron dense nucleus and are membrane bound (arrows). Scale bar $2 \mu \mathrm{m}$.

VSMCs (controls $0 \%, \mathrm{p}<0.001$ ) and, as confirmed by double labeling, localised to the cytoplasm of smooth muscle cells ( $\alpha$-actin positive) bordering the plane of dissection, the cystic areas in the media, and to the vicinity of the vasa vasorum in the outer part of the

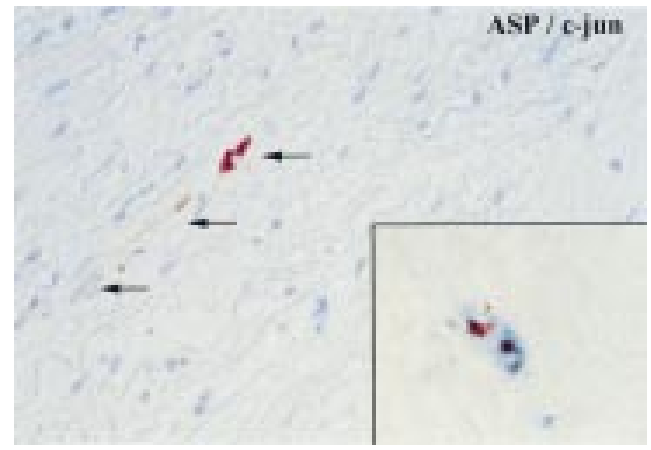

Figure 7 Section of a human aorta with CMD showing few medial VSMCs with a positive cytoplasmic staining for c-jun/ASP (brown reaction product). Inset: double labeling showing a medial VSMCs with a positive TUNEL stain in the nucleus (brown reaction product) and cytoplasmic staining for c-jun/ASP (blue reaction product, $A P A A P$ method). The TUNEL positive nucleus shows a fragmentation which is a typical morphological sign of apoptosis.

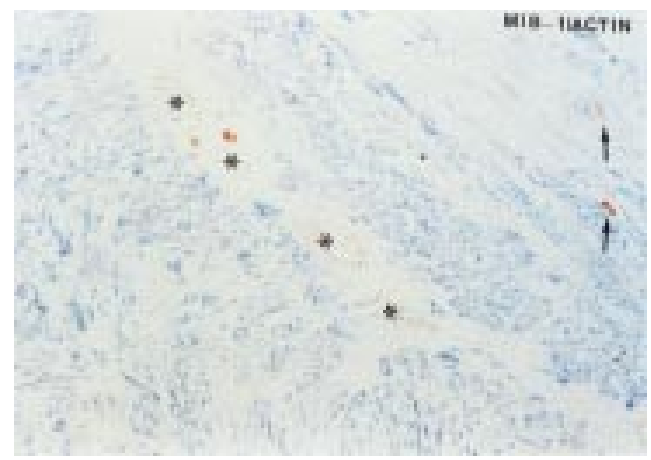

Figure 8 Double immunostaining for MIB1/Ki-67 (brown reaction product, $A B C$ method) and a-actin (blue reaction product, APAAP method) showing three MIB1/Ki-67 positive/a-actin negative cells in the regions around the vasa vasorum (asterisks) and two MIB1/Ki-67 positive/a-actin positive VSMCs in the media (arrows).

media. In contrast, in the controls, c-jun staining was absent. Moreover, and confirming recent observations by Björkerud, ${ }^{25} \mathrm{ASP} / \mathrm{c}-$ jun-IR was present in scattered intimal cells in $12 / 20$ cases. Importantly, as shown by serial sections, ASP/c-jun-IR colocalised with p53-IR in all $20 \mathrm{ASP} / \mathrm{c}$-jun positive cases in medial smooth muscle cells. In addition, as shown by double labeling, we found few TUNEL positive cells showing at the same time intense cytoplasmic staining for $\mathrm{ASP} / \mathrm{c}-$ jun (fig 7 inset).

MIB1/KI-67 IMMUNOHISTOCHEMISTRY

MIB1/Ki-67-IR is localised mainly in the regions around the vasa vasorum (20/20 cases, fig 8 asterisks), but to a far lesser extent in scattered cells in the media (fig 8, arrows) and around the cystic spaces. As confirmed by double immunolabeling, these cells were either $\alpha$-actin positive smooth muscle cells or $\alpha$-actin negative/vimentin positive mesenchymal cells. MIB1/Ki-67-IR indicating proliferative activity was identified in all 20 specimens in 2.5 (2.8)\% of the cells, whereas in the control group MIB1/Ki-67-IR was present in $0.3(0.9) \%$ $(p<0.001)$. Interestingly, despite the focal disappearance of nuclei, the average cell numbers per high power field in the aortic tissue with CMD were higher than those in the 
control tissue (125 (55); controls 79 (31), $\mathrm{p}<0.001)$

\section{Discussion}

Apoptosis is a selective process of physiological cell deletion, also known as programmed cell death. ${ }^{28}{ }^{29}$ Programmed cell death is a genetically controlled response of individual cells that are no longer needed, to commit suicide. It generally involves changes in the expression of proapoptotic and antiapoptotic genes and gene products including both the tumour suppressor gene p53 as well as the proapoptotic protein of the Bcl2 family Bax. ${ }^{73}$ Recent studies suggest that impaired regulation of apoptosis plays a pivotal role in the pathogenesis of both neoplastic and degenerative diseases including cardiovascular disorders. ${ }^{30-32} \mathrm{CMD}$ is a degenerative disease of the aortic media characterised by profound changes in the extracellular matrix leading to structural inhomogeneity, enhanced circumferential wall stress and shear stress, ${ }^{21}$ aneurysm formation, and focal loss of VSMCs. ${ }^{2}$ However, little is known about the cellular reactions during CMD. The present study was designed to evaluate the hypothesis that p53 accumulation, Bax upregulation, programmed cell death, and tissue repair processes are involved in the mechanisms underlying CMD of the aorta. Our results show for the first time that CMD is characterised by the presence of significant numbers of medial VSMCs with nuclear p53 accumulation, cytoplasmic Bax expression, and signs of apoptosis. In addition, as shown by $\mathrm{MIB} 1 / \mathrm{Ki}-67^{33}$ expression, we have found significant numbers of medial VSMCs which were in the late G1 or more advanced stages of the cell cycle together with a significantly higher cell count per high power field, indicating that at least a proportion of the MIB1/Ki-67 positive cells in fact proliferate. Therefore, the results of the present study show that CMD bears characteristics of a "response to injury process".

Apoptosis of smooth muscle cells was confirmed by several independent complementary techniques including TUNEL labeling, biochemical DNA fragmentation analysis, electron microscopy, and ASP/c-jun staining. In the absence of any inflammation, we observed scattered TUNEL positive and ASP/c-jun positive apoptotic smooth muscle cells in the vicinity of the morphologically characteristic mucopolysaccharide filled small pseudocysts and the areas surrounding the vasa vasorum, which is a crucial feature of programmed cell death, because it permits cell removal without damage to adjacent cells. ${ }^{5}{ }^{6}$ Since apoptosis, once initiated, is a very quick process, the overall level of apoptosis in tissue sections can only be roughly quantified. However, in the present study, the number of apoptotic cells was $1.01 \%$ of the medial smooth muscle cells.

Most TUNEL positive, ASP/c-jun positive, p53 positive, and Bax positive cells were located in a homogeneous distribution in the immediate vicinity of the dissection plane, whereas the distribution of these cells in the areas which were not affected by the dissection was random and patchy. This may give rise to the assumption that apoptotic mechanisms underlie the dissection process. However, as we do not know the exact time course of the dissection - be it acute, subacute, or chronicthe demonstration of cells with p53 accumulation, Bax expression, and with signs of apoptosis in the region around the plane of dissection may be the morphological substrate of a reactive process caused by a sublethal injury of cells by the dissection itself.

The morphological alterations of CMD are considered to be non-specific, occurring during the normal aging of the aorta. ${ }^{34}$ Nevertheless, these changes are more widespread in patients with an altered haemodynamic profile. ${ }^{4}$ They encompass degeneration of the extracellular matrix and destruction of the elastic fibre system, which can no longer absorb the mechanical forces imposed on the tissue during systole. This probably leads to the occurrence of considerable shear forces acting on the medial VSMCs and may predispose to dissection. ${ }^{21}{ }^{34} \mathrm{CMD}$ may thus be regarded as an in vivo model to study the effects of enhanced mechanical forces on medial VSMCs. Therefore, confirming recent in vitro observations about the effects of stretch mediated reactions on cardiac myocyte and nonmyocyte cells, ${ }^{22}{ }^{35}{ }^{36}$ the demonstration of medial VSMCs with p53 accumulation, Bax expression, and cytoplasmic staining for ASP/ c-jun, as well as apoptotic cells mainly in regions with uneven distribution of circumferential wall stress (for example, the vicinity of cystic spaces and the regions around the vasa vasorum), may indicate that mechanical stress may be a possible cause of p53 accumulation, Bax upregulation and/or apoptosis of VSMCs during CMD. However, the precise mechanisms of the upregulation of both proapoptotic proteins still remain elusive. In addition, the profound disturbances in the local environment of the medial VSMCs in the tissue with CMD (for example, the destruction of the extracellular matrix) may also play an important role in the regulation of apoptosis and influence cellular regeneration during CMD. ${ }^{37} 38$

We observed p53 positive/Bax positive cells with VSMC like morphology giving rise to the assumption that in a part of the $\mathrm{p} 53$ positive cells the Bax gene is transactivated by $\mathrm{p} 53$. However, the colocalisation of both proteins does not prove a cause and effect relation. In addition, the detection of p53 negative/Bax positive cells may indicate that Bax expression occurs also independently from $\mathrm{p} 53$.

Moreover, we have found p53 accumulation and Bax expression in a very small number of medial smooth muscle cells in the control tissue which exhibited at the same time minor degrees of CMD. In contrast, in the aortic tissue from patients with higher degrees of CMD, p53 as well as Bax staining were widespread in a patchy and irregular distribution, indicating that $\mathrm{p} 53$ accumulation and Bax expression not only correlate with the severity of CMD but may rather be a reactive phenomenon during CMD. 
We have found cell death rates of about $1 \%$ of the total cell number in the specimens with CMD. On the other hand, the total cell numbers in the tissue with CMD were significantly higher compared to those of the control tissue. Thus, we may safely assume that in the tissue with CMD there must be a certain degree of cell proliferation. This may be indicative of an ongoing disorganised regeneration process leading to the formation of a disorderly arranged medial tissue with reduced functional properties.

Finally, it has recently been shown that p53, Bax, and programmed cell death are involved in the regulation of cell turnover in human carotid atherosclerotic plaques and in human aortic atherosclerotic abdominal aneurysms with chronic inflammation. ${ }^{1526}$ The results of our study go considerably beyond these previous observations by showing that $\mathrm{p} 53$, Bax, and programmed cell death also play a significant role during CMD of the aorta which is, in contrast to atherosclerosis, an entirely noninflammatory and degenerative disease of the aorta with a completely different pathogenetic background.

In conclusion, we have shown that the formal pathogenesis of CMD of the aorta is characterised by p53 accumulation, Bax upregulation, cell death by apoptosis, and tissue repair and, therefore, exhibits features of a response to injury process as already stated earlier by Schlattmann and Becker. ${ }^{3}$ However, the precise mechanisms of $\mathrm{p} 53$ and Bax upregulation as well as the role of $\mathrm{p} 53$ and apoptosis in the dissection process itself remain elusive.

We are grateful to Professor AE Becker for suggestions concerning the preparation of this manuscript. Dr Nampoothiri was supported by a grant from the International Atherosclerosis Society, Houston, Texas, USA, and Dr Szombathy was supported by a grant from the University of Freiburg, Germany. We thank Gabriele von Kürthy, Adelheid Federspiel, Marlies Thome, and Hildegard Metzger for their skilful technical assistance.

1 Larson EW, Edwards WD. Risk factors for aortic dissection: a necropsy study of 161 cases. Am $\mathcal{F}$ Cardiol 1984;53:84955 .

2 DeSanctis RW, Doroghazi RM, Austen WG, et al. Aortic dissection. N Engl F Med 1987;317:1060-7.

3 Schlattmann TJM, Becker AE. Histologic changes in the normal aging aorta: implications for dissecting aortic aneurysm. Am f Cardiol 1977;39:13-20.

4 Schlattmann TJM, Becker AE. Pathogenesis of dissecting aneurysm of aorta: comparative histopathologic study of significance of medial changes. Am F Cardiol 1977;39:21-6.

5 Majno G, Joris I. Apoptosis, oncosis, and necrosis: an overview of cell death. Am 7 Pathol 1995;146:3-15

6 Haunstetter A, Izumo S. Apoptosis: basic mechanisms and implications for cardiovascular disease. Circ Res 1998;82 $1111-29$

7 Oltvai Z, Milliman CL, Korsmeyer SJ. Bcl-2 heterodimerizes in vivo with a conserved homolog, Bax, that accelerates programmed cell death. Cell 1993;74:609-19.

8 Miyashita T, Reed J. Tumor suppressor p53 is a direct transcriptional activator of the human Bax gene. Cell 1995;80: 293-9.

9 Miyashita T, Harigai M, Hanada M, et al. Identification of a p53-dependent negative response element in the bcl-2 p53-dependent negative response
gene. Cancer Res 1994;54:3131-5.

10 Kastan MB, Zhan Q, El-Deiry WS, et al. A mammalian cell cycle checkpoint pathway utilizing p53 and GADD45 is defective in ataxia-telangiectasia. Cell 1992;71:587-97.
11 Rotter V, Aloni GR, Schwartz D, et al. Does wild-type p53 play a role in normal cell differentiation? Semin Cancer Biol play a role in no:

12 Gottlieb TM, Oren M. p53 in growth control and neoplasia. Biochem Biophys Acta 1996;287:77-102.

13 Oren M. Relationship of p 53 to the control of apoptotic cell death. Semin Cancer Biol 1994;5:221-7.

4 Bennett MR, Littlewood DT, Schwartz SM, et al. Increased sensitivity of human vascular smooth muscle cells from atherosclerotic plaques to p53-mediated apoptosis. Circ Res 1997;81:591-9.

15 López-Candales A, Holmes DR, Liao S, et al. Decreased vascular smooth muscle cell density in medial degeneration of abdominal

16 Ihling C, Haendeler J, Menzel G, et al. Coexpression of p53 and MDM2 in human atherosclerosis: implications for the regulation of cellularity of atherosclerotic lesions. F Pathol 1998;185:303-12.

17 Ihling C, Menzel G, Wellens E, et al. Topographical association between the cyclin-dependent kinases inhibitor p21, p53 accumulation, and cellular proliferation in human atherosclerotic tissue. Arterioscler Thromb Vasc Biol 1997;17: 2218-24

8 Kuwano K, Hagimoto N, Nomoto Y, et al. p53 and p21 (Waf1/Cip1) mRNA expression associated with DNA damage and repair in acute immune complex alveolitis in mice. Lab Invest 1997;76:161-9.

19 Kuwano K, Kunitake R, Kawasaki M, et al.p21(Waf1/Cip1) and p53 expression in association with DNA strand breaks in idiopathic pulmonary fibrosis. Am f Respir Crit Care Med 1996;154:477-83.

20 Antoniades HN, Galanopoulos T, Nevile-Golden J, et al. p53 expression during normal tissue regeneration in response to acute cutaneous injury in swine. 7 Clin Invest 1994;93:2206-14.

21 Davies MJ, Treasure T, Richardson P. The pathogenesis of arterial dissection. Heart 1996;75:434-5.

22 Leri A, Claudio PP, Li Q, et al. Stretch-mediated release of angiotensin II induces myocyte apoptosis by activating p53 that enhances the local renin-angiotensin-system and decreases the Bcl-2-to-Bax protein ratio in the cell. $f$ Clin Invest 1998;101:1326-42.

23 Kockx MM, Muhring J, Knaapen MW, et al. RNA synthesis and splicing interferes with DNA in situ end labeling techniques used to detect apoptosis. Am F Pathol 1998;152: $885-8$.

24 Grand RJA, Milner AE, Mustoe T, et al. A novel protein expressed in mammalian cells undergoing apoptosis. Exp Cell Res 1995;218:439-51.

25 Björkerud S, Björkerud B. Apoptosis is abundant in human atherosclerotic lesions, especially in inflammatory cells (macrophages and $\mathrm{T}$ cells), and may contribute to the accumulation of gruel and plaque instability. Am 7 Pathol 1996;149:367-80.

26 Kockx MM, DeMeyer GRY, Muhring J, et al. Apoptosis and related proteins in different stages of human atherosclerotic plaques Circulation 1998:97:2307-15.

27 Stary HC, Chandler AB, Glagov S, et al. A definition of initial, fatty streak, and intermediate lesions of atherosclerosis: a report from the committee on vascular lesions of the council on atherosclerosis, American Heart Association. Circulation 1994;89;2462-78.

28 Kerr JFR. Shrinkage necrosis: a distinct mode of cellular death. F Pathol 1971;105:13-21.

29 Steller H. Mechanisms and genes of cellular suicide. Science 1995;267:1445-9.

30 Carson DA, Ribeiro JM. Apoptosis and disease. Lancet 1993;341:1251-4.

31 Isner JM, Kearney M, Bortmann S, et al. Apoptosis in human atherosclerosis and restenosis. Circulation 1995;91: 2703-11.

32 James TN. Normal and abnormal consequences of apoptosis in the human heart: from postnatal morphogenesis to paroxysmal arrythmias. Circulaion 1994;90:556-73.

33 Gerdes J, Li L, Schlueter C, et al. Immunobiochemical and molecular biologic characterization of the cell proliferationassociated nuclear antigen that is defined by monoclonal antibody Ki-67. Am F Pathol 1991;138:867-73.

34 Richardson P, Davies M, Born G. Influence of plaque configuration and stress distribution on fissuring of coronary atherosclerotic plaques. Lancet 1989;ii:941-4.

35 Cheng W, Li B, Kajstura J, et al. Stretch-induced programmed myocyte cell death. F Clin Invest 1995;96: 2247-59.

36 Teiger E, Dam TV, Richard L, et al. Apoptosis in pressure overload-induced heart hypertrophy in the rat. $\mathcal{F}$ Clin Invest 1996;97:2891-7.

37 Ruoslahti E, Reed J. Anchorage dependence, integrins, and apoptosis. Cell 1994;77:477-8.

38 Assoian RK, Marcantonio EE. The extracellular matrix as a cell cycle control element in atherosclerosis and restenosis. f Clin Invest 1996;98:2436-9. 